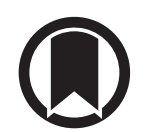

CrossMark

\title{
A musical intervention for respiratory comfort during noninvasive ventilation in the ICU
}

\author{
Jonathan Messika (1) 1,2,3, Yolaine Martin', Natacha Maquigneau4 \\ Christelle Puechberty ${ }^{5}$, Matthieu Henry-Lagarrigue ${ }^{4}$, Annabelle Stoclin ${ }^{5}$, \\ Nataly Panneckouke ${ }^{1}$, Serge Villard ${ }^{1}$, Aline Dechanet ${ }^{6}$, Alexandre Lafourcade ${ }^{7}$, \\ Didier Dreyfuss ${ }^{1,2,3}$, David Hajage ${ }^{8,9}$ and Jean-Damien Ricard ${ }^{1,2,3}$, on behalf of \\ the MUS-IRA team ${ }^{\text {io }}$
}

Affiliations: ${ }^{1}$ Medical-Surgical Intensive Care Unit, Hôpital Louis Mourier, AP-HP, Colombes, France. ${ }^{2}$ UMRS 1137 (IAME), Sorbonne Paris Cité, Université Paris Diderot, Paris, France. ${ }^{3}$ UMRS 1137 (IAME), INSERM, Paris, France. ${ }^{4}$ Medical-Surgical Intensive Care Unit, District Hospital Center, La Roche-sur-Yon, France. ${ }^{5}$ Medical-Surgical Intensive Care Unit, Institut Gustave Roussy, Villejuif, France. ${ }^{6}$ Unité de Recherche Clinique Paris-Nord, Hôpital Bichat, AP-HP, Paris, France. ${ }^{7}$ Dépt de Biostatistiques, Santé Publique et Information Médicale, Hôpital Pitié-Salpêtrière, AP-HP, Paris, France. ${ }^{8}$ Dépt de Biostatistique, Santé Publique et Information Médicale, Centre de Pharmaco-Epidémiologie de l'AP-HP, Sorbonne Université, CIC 1421, Hôpital Pitié-Salpêtrière, AP-HP, Paris, France. ${ }^{9}$ UMRS 1123 (ECEVE), CIC-EC 1425, INSERM, Paris, France. ${ }^{10} \mathrm{~A}$ list of the MUS-IRA investigators can be found at the end of this article.

Correspondence: Jonathan Messika, Medical-Surgical Intensive Care Unit, Hôpital Louis Mourier, 178 Rue des Renouillers, F-92700, Colombes, France. E-mail: jonathan.messikadaphp.fr

@ERSpublications

A 30-min musical intervention in ARF patients requiring NIV decreases peri-traumatic symptoms at ICU discharge http://ow.ly/UVOE30myIbM

Cite this article as: Messika J, Martin Y, Maquigneau N, et al. A musical intervention for respiratory comfort during noninvasive ventilation in the ICU. Eur Respir J 2019; 53: 1801873 [https://doi.org/10.1183/ 13993003.01873-2018].

ABSTRACT Discomfort associated with noninvasive ventilation (NIV) may participate in its failure. We aimed to determine the effect of a musical intervention on respiratory discomfort during NIV in patients with acute respiratory failure (ARF).

An open-label, controlled trial was performed over three centres. Patients requiring NIV for ARF were randomised to either a musical intervention group (where they received a musical intervention and were subjected to visual deprivation during the first $30 \mathrm{~min}$ of each NIV session), a sensory deprivation group (where they wore insulating headphones and were subjected to visual deprivation during the first $30 \mathrm{~min}$ of each NIV session), or a control group (where they received NIV as routinely performed). The primary outcome was the change in respiratory discomfort before and after $30 \mathrm{~min}$ of the first NIV session.

A total of 113 patients were randomised (36 in the musical intervention group, 38 in the sensory deprivation group and 39 in the control group). Median (interquartile range (IQR)) change in respiratory discomfort was $0(-1 ; 1)$ between the musical intervention and control groups $(p=0.7)$. Between groups comparison did not evidence any significant variation of respiratory parameters across time or health-related quality of life (HRQoL) at day-90. The Peri-traumatic Distress Inventory (PDI) at intensive care unit (ICU) discharge was reduced in musical intervention group patients. However, a $30 \mathrm{~min}$ musical intervention did not reduce respiratory discomfort during NIV for ARF in comparison to conventional care or sensory deprivation.

This article has supplementary material available from erj.ersjournals.com

Received: July 152018 | Accepted after revision: Oct 312018

This study is registered at ClinicalTrials.gov with identifier NCT02265458. Individual data (including data dictionaries) that underlie the results reported in this article will be shared after de-identification (text, tables, figures and appendices). Additional, related documents (e.g. study protocol and statistical analysis plan) will be available. Data will become available immediately after article publication and for a period of 5 years. Anyone who wishes to access the data for any purpose should submit a data sharing proposal to the corresponding author. 


\section{Introduction}

Noninvasive ventilation (NIV) use in acute respiratory failure (ARF) is known to reduce intubation rates, nosocomial infections [1] and mortality [2] in some critical care situations [3, 4]. Among the drawbacks of NIV is poor tolerance [5], which is known to increase the risk of intubation [6-8]. In addition, the setting and conducting of NIV is time-consuming and generates a high level of stress in the patient, their relatives and their caregivers [9]. Among the pharmacological strategies to enhance patients' tolerance to NIV, sedation has been shown to reduce NIV-induced discomfort [10-13]. Nevertheless, these anesthetic treatments may be dangerous if not used cautiously and require additional precautions by trained staff.

Among the non-pharmacological interventions available, music therapy is increasingly being investigated [14]. In patients undergoing invasive mechanical ventilation, music therapy facilitates a reduction in anxiety and sedation exposure in comparison with conventional strategies or sensory deprivation [15]. Furthermore, significant changes in physiologic variables have been evidenced $[16,17]$. These positive findings led us to conduct a trial to ameliorate the tolerance of a stressful intensive care unit (ICU) technique [9]. We therefore aimed to assess the potential benefit of music therapy as a non-pharmacological adjunct to NIV to enhance acceptance and tolerance of the technique. As "music therapy" implies the intervention of a qualified music therapist, we chose to use the term "musical intervention", which refers to a receptive music session administered to a patient by trained caregivers. In order to distinguish between the effect of music and that of noise, and because no side sensory deprivation had been described [18], we also chose to test the sensory deprivation strategy.

\section{Methods}

\section{Study design and participants}

The MUSique pour l'Insuffisance Respiratoire Aigue (Mus-IRA) trial is a prospective, multicentre, open-label, three-arm randomised trial [19]. Patients were enroled from May 2015 to June 2016 in three French adult ICUs that already provided patient-centred care [19]. Written informed consent was obtained from all patients. Patients older than 18 years with ARF and a sufficient level of consciousness (Glasgow Coma Scale (GCS) score $\geqslant 12$ ) were enroled if they required NIV. Non-inclusion criteria were a severe hearing impairment (a classical contra-indication to NIV), a decision to withdraw life-sustaining therapies with an estimated life expectancy of less than $48 \mathrm{~h}$, or participation in another trial dealing with ARF. The protocol was approved by the Comité de Protection des Personnes Paris-Ile-de-France IV (No. 2014-A00643-44) and the ethics committee of French Society for Intensive Care Medicine (Société de Réanimation de Langue Française, approval CE SRLF 14-21).

\section{Randomisation and masking}

All procedures are summarised in the supplementary material. Briefly, eligible participants were randomly allocated to one of the three study arms in a 1:1:1 ratio via a computer-generated, interactive web-response system (Cleanweb, Telemedecine Technologies S.A.S, Boulogne-Billancourt, France). After having removed and stored all the material, the primary outcome was blindly assessed by a nurse or a nurse-assistant from another unit.

\section{Procedures}

After inclusion, all participants underwent NIV in a similar manner according to standards of care and in-line with each participating ICUs practices (as described in the supplementary material). Once an NIV mask was fitted and the ventilator settings were optimised, participants allocated to the musical intervention group had dedicated headphones (BOSE AE2, BOSE Corp, Purmerend, The Netherlands) positioned over their ears. They were shown how to handle the tablet interface (Samsung Galaxy, Samsung Electronics Co, Korea) by the trained nurse or nurse-assistant and their musical tastes were determined by a caregiver-administered questionnaire. The patients chose their musical program according to their preferences, set the volume level and began a 30-min "L-type" musical intervention session (MUSIC CARE, Paris, France), as described in the supplementary material. A sleeping mask was then applied to cover their eyes. In the sensory deprivation group, once the NIV session was initiated the same insulating headphones (BOSE AE2) were placed on the patients' ears (without any music) and their eyes were covered by a sleeping mask. In both intervention groups, the headphones and masks were left in place for $30 \mathrm{~min}$. In the control group, NIV was conducted as usually performed in the participating units. All subsequent NIV sessions were conducted according to the randomisation group until NIV discontinuation, day-28 or ICU discharge. In all three randomisation arms any other treatments were left to the discretion of the treating physicians.

The patient rated their respiratory discomfort with the use of a visual scale. They were asked to rate the intensity of their respiratory discomfort on a $10-\mathrm{cm}$ long ruler shaped like an arrow. It was bounded by "0: no respiratory discomfort" (the smallest base of the arrow) and to the right "10: maximal respiratory 
discomfort" (the head of the arrow). Patients marked directly on the ruler the level of their perception of discomfort. This measure was assessed prior to each NIV session, immediately after NIV was correctly set (5 min), at $30 \mathrm{~min}$ and at $1,2,3,4,6,8,12,16,20$, and $24 \mathrm{~h}$ according to NIV session length. Quality of life was determined using the Hospital Anxiety and Depression Scale (HADS) [20] and 36-item Short-Form Health Survey (SF-36) [21] questionnaires at baseline and at day-90. On ICU discharge and at day-90, the research team submitted to the participants the Peri-traumatic Distress Inventory (PDI) [22, 23] applied to NIV, as well as a numerically scaling survey of NIV satisfaction, discomfort and NIV-associated trauma.

\section{Objectives and outcomes}

We aimed to assess the hypothesis that a musical intervention administered to ICU patients with ARF would reduce respiratory discomfort and thereby improve NIV tolerance and ventilation parameters after $30 \mathrm{~min}$ of NIV (in comparison to conventional care or to isolation from the ICUs noise and light nuisance). We also aimed to assess the effect of musical intervention on physiological parameters, NIV failure and tolerance, and patient-centred outcomes such as anxiety, depression, peri-traumatic stress and NIV appreciation.

The primary endpoint was the change in respiratory discomfort between the time before initiation and after $30 \mathrm{~min}$ of the first NIV session after randomisation. Secondary endpoints were: 1) evolution of respiratory discomfort during the first NIV session and at the end of the session (and similarly for each subsequent NIV session); 2) changes in respiratory parameters during NIV sessions (respiratory rate, transcutaneous oxygen saturation and exhaled tidal volume); 3) changes in cardiovascular parameters during NIV sessions (heart rate and arterial pressure) with all endpoints being assessed at the same time-points (see above and in the supplementary material for details); 4) percentage of patients requiring endotracheal intubation (NIV failure) at the end of a NIV session; 5) agreement between the duration of the prescribed and the actually delivered NIV session; 6) number of sessions interrupted before the end of the prescribed time; 7) percentage of patients requiring physical restraint or sedative/anxiolytic treatments during NIV sessions and ICU stay; 8) anxiety/depression and health-related quality of life (HRQoL) measured by HADS and SF-36 scores at baseline and at day-90; 9) peri-traumatic stress measured with the PDI immediately on ICU discharge and at day-90; and 10) patients' overall assessment of NIV (in terms of discomfort, satisfaction and trauma) at ICU discharge and day-90.

In order to explore musical intervention effects, some other outcomes were assessed in a post-hoc analysis, as follows: 1) NIV tolerance was assessed based on the number of NIV sessions with an attempt to pull off the NIV interface and the relative risk of premature interruption of the NIV session; 2) agitation was assessed based on its evolution by the Richmond Agitation-Sedation Scale (RASS); and 3) efficacy was assessed by the duration of NIV treatment in days, the number of NIV sessions per patient and survival at ICU discharge.

\section{Statistics}

The sample size was calculated a priori as detailed in the supplementary material and it was expected that a total of 99 participants (33 per arm) would be randomised. In May 2016, after the inclusion of 80 subjects, a blinded quality assessment of the study data (which did not involve any comparison of study outcomes between groups) showed that the primary endpoint had not been collected for 14 participants (who were asleep under NIV after the first $30 \mathrm{~min}$ of therapy). The number of subjects randomised was therefore increased to 113 .

Continuous data are presented as median (25\%-75\% interquartile range (IQR)) unless otherwise specified. Dichotomous data are presented as number and percentage. All analyses were performed on the intentionto-treat population. Unless otherwise specified, categorical variables were compared by a Chi-squared test or a Fisher test and continuous variables were compared by a $t$-test or a Wilcoxon test (when two groups are compared), or an ANOVA test or a Kruskal-Wallis test (when more than two groups are compared). The analyses were performed with SAS, version 9.4 (SAS Institute, Cary, NC, USA) and further details are given in the supplementary material.

\section{Results}

\section{Patients}

Between May 2015 and June 2016, 311 consecutive patients were screened in the three participating ICUs. A total of 113 were included in the trial and randomised, such that 39 were allocated to the control group, 36 to the musical intervention group and 38 to the sensory deprivation group (figure 1). Baseline characteristics of the three groups are shown in table 1 . The main reason for NIV initiation was acute-onchronic respiratory failure $(\mathrm{n}=61 ; 54 \%)$. 


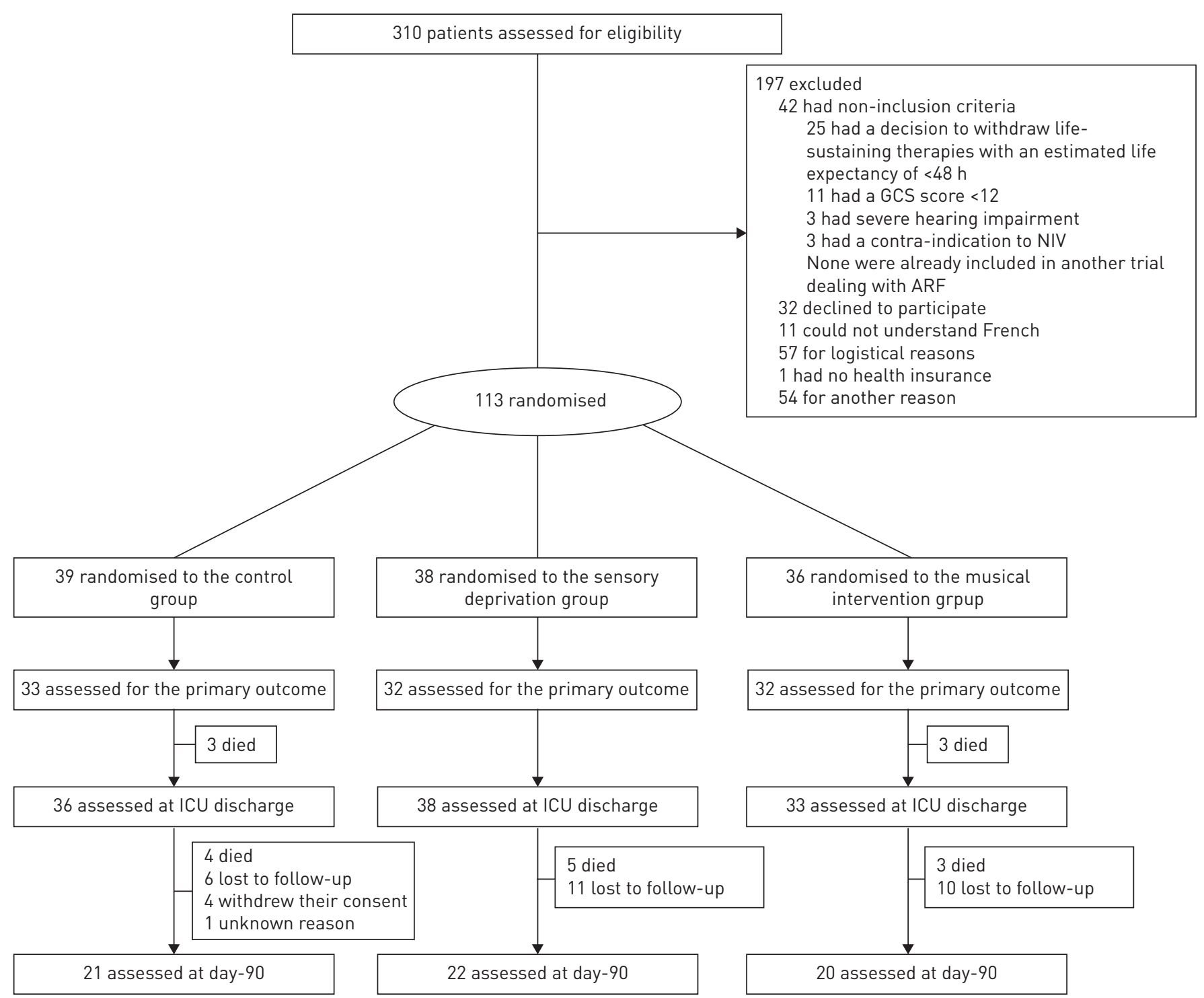

FIGURE 1 Flow diagram for inclusion, randomisation and assessment of patients. Other reasons for non-inclusion were: noninvasive ventilation (NIV) refusal, NIV for pre-oxygenation, NIV interruption after a single session, legal impairment for inclusion, patient under the age of 18 and patient already included in the study. The primary outcome could not be assessed for some of the subjects as they were asleep under NIV after the first 30 min of therapy. ICU: intensive care unit; GCS: Glasgow Coma Scale; ARF: acute respiratory failure.

\section{Tolerance of musical intervention and sensory deprivation}

During the first NIV session, 15 out of 38 patients (39.5\%) in the sensory deprivation group complied with the protocol and accepted both visual and auditory deprivation. However, nine patients $(23.7 \%)$ rejected both visual and auditory deprivation, nine (23.7\%) rejected visual deprivation only and two (5.3\%) rejected auditory deprivation only. All musical intervention patients accepted the musical intervention and 14 out of $36(38.9 \%)$ of those accepted the visual deprivation

\section{Primary and secondary outcomes}

Details on primary and secondary outcomes are presented in table 2 and table 3 . The change in respiratory discomfort from before initiation to after $30 \mathrm{~min}$ of the first NIV session after randomisation was not different between groups, with a median difference of $-1(-2 ; 0)$ for the control group, $0(-2 ; 0)$ for the sensory deprivation group and $-1(-3 ; 1.1)$ for the musical intervention group ( $\mathrm{p}=0.7$ by Wilcoxon rank-sum test) (figure 2). Sensitivity analyses of the primary outcome were performed (adjustment for stratification factors, potential confounders, imputation of missing values and per-protocol analysis) and no significant differences in the change in respiratory discomfort were evidenced. Variation in respiratory discomfort remained non-significantly different between subsequent time-points and for subsequent sessions. 
TABLE 1 Baseline data for the study

Group

\begin{tabular}{|c|c|c|c|}
\hline & & & \\
\hline & Control $(n=39)$ & Sensory deprivation $(n=38)$ & Musical intervention $(\mathrm{n}=36)$ \\
\hline Male gender & $18(46.2)$ & $21(55.3)$ & $23(63.9)$ \\
\hline SAPS $\|^{\#}$ score & $35.5(27-47)$ & $32(24-40)$ & $35(27-41)$ \\
\hline \multicolumn{4}{|l|}{ Comorbid conditions } \\
\hline Chronic cardiopathy & $5(12.8)$ & $8(21.1)$ & $2(5.6)$ \\
\hline Chronic respiratory disease & $30(76.9)$ & $25(65.8)$ & $22(61.1)$ \\
\hline Alcohol consumption & $4(10.3)$ & $9(23.7)$ & $10(27.8)$ \\
\hline Smoker or ex-smoker & $28(71.8)$ & $24(63.2)$ & $27(75)$ \\
\hline \multicolumn{4}{|l|}{ HRQoL } \\
\hline HADS score & $16(12-23)$ & $17(15-19.5)$ & 15 (13-19) \\
\hline Anxiety sub-score & $8.5(6-12)$ & $8.5(5.5-10.5)$ & $8(6-10)$ \\
\hline Depression sub-score & $8(5-10)$ & $8(7-11)$ & $7(5-9)$ \\
\hline Acute-on-chronic respiratory failure & $24(61.4)$ & $19(50)$ & $18(50)$ \\
\hline Pulmonary oedema & $10(25.6)$ & $11(28.9)$ & $10(27.8)$ \\
\hline De novo respiratory failure & $6(15.4)$ & $8(21.1)$ & $7(19.4)$ \\
\hline Post-extubation respiratory failure & $2(5.1)$ & $3(7.9)$ & $3(8.3)$ \\
\hline Post-operative respiratory failure & $3(7.7)$ & $3(7.9)$ & $5(13.9)$ \\
\hline Chest trauma & $0(0)$ & $2(5.3)$ & $0(0)$ \\
\hline Post-extubation respiratory failure prophylaxis & $0(0)$ & $2(5.3)$ & $1(2.8)$ \\
\hline \multicolumn{4}{|l|}{ ICU therapies prior to enrolment } \\
\hline Invasive mechanical ventilation & $3(7.7)$ & $5(13.2)$ & $6(16.7)$ \\
\hline Noninvasive mechanical ventilation & $18(46.2)$ & $22(57.9)$ & $14(38.9)$ \\
\hline GCS score at enrolment & $15(15-15)$ & $15(15-15)$ & $15(15-15)$ \\
\hline Respiratory rate breaths. $\min ^{-1}$ & $25(20-29)$ & $24(21.5-28)$ & $24(20-27)$ \\
\hline
\end{tabular}

In a similar manner, variation in respiratory parameters over time was not significantly different between groups. Notably, a significant reduction in systolic and mean arterial pressure was evidenced in the musical intervention group for the first NIV session; however, no significant variation of these parameters was observed for subsequent sessions. NIV failure rates did not differ significantly between groups $(7.7 \%$ (control group) versus $2.6 \%$ (sensory deprivation group) versus $0 \%$ (musical intervention group), $\mathrm{p}=0.32$ ).

HRQoL at day-90 did not differ between groups with regard to the anxiety and depression sub-scores of the HADS or the physical and mental dimensions of the SF-36. However, on ICU discharge, PDI was significantly reduced $(\mathrm{p}=0.03)$ in musical intervention group patients (PDI $8(5-13)$ ) versus sensory deprivation patients (PDI 16 (5-23)) and control group patients (PDI 16 (12-25)). This difference was no longer significant at day-90. Other outcomes are detailed in the supplementary material.

\section{Discussion}

This prospective randomised controlled assessment of a musical intervention during NIV for ARF in ICU patients failed to evidence a significant reduction in respiratory discomfort in comparison to conventional care. However, it did show a significant decrease in systolic and mean arterial blood pressure (BP) at the 
TABLE 2 Clinical outcomes in the study

Control ( $n=39) \quad$ Sensory deprivation ( $n=38$ ) Musical intervention ( $n=36)$

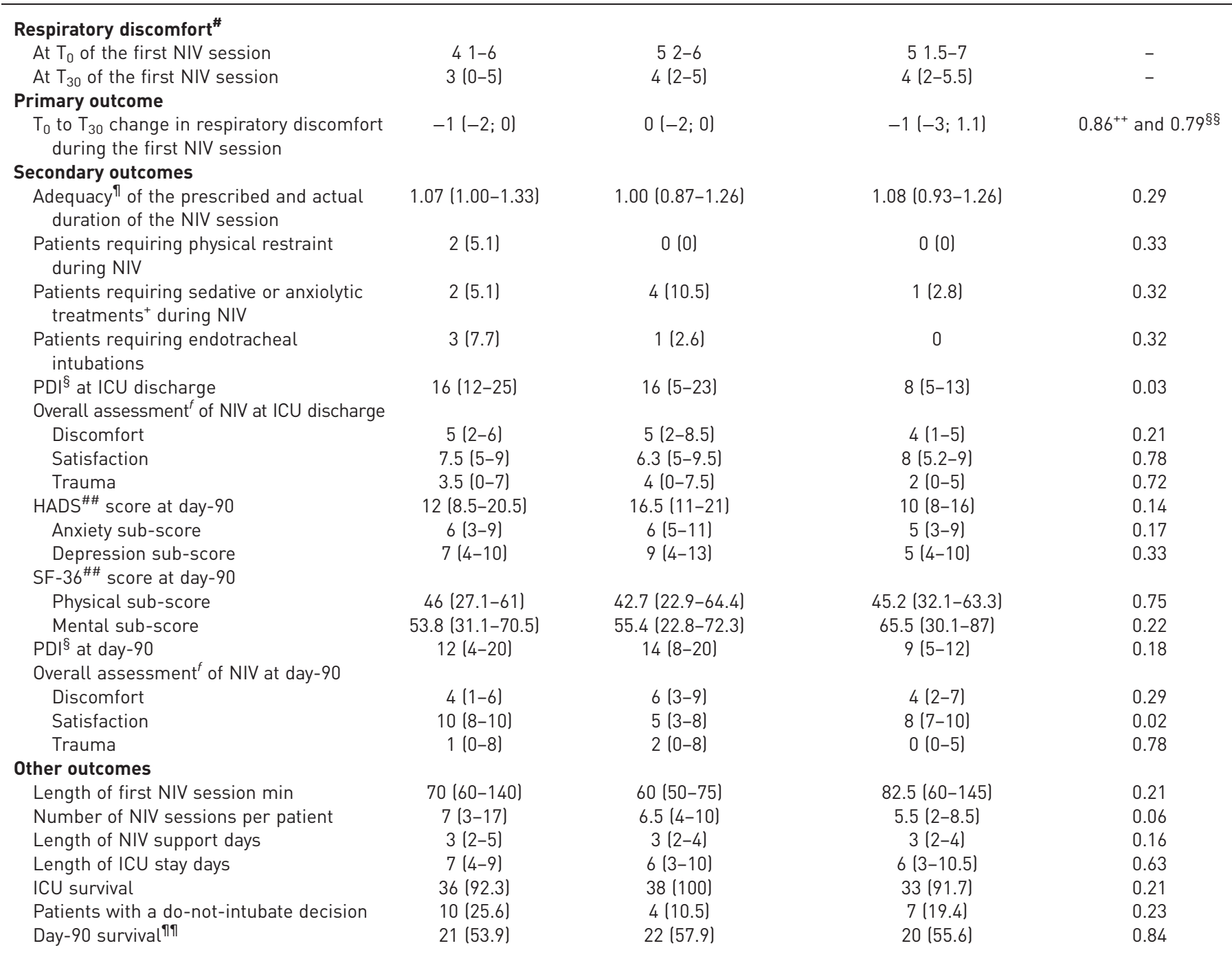

Data is presented as $\mathrm{n}(\%)$ or median (IQR). p-Values measure the comparison between the three groups unless otherwise stated. NIV: noninvasive ventilation; $T_{0}$ : time of assessment of respiratory discomfort prior to each NIV session; $T_{30}$ : time of the assessment of respiratory discomfort after 30 min of each NIV session; PDI: Peri-traumatic Distress Inventory; ICU: intensive care unit; HADS: Hospital Anxiety and Depression Scale; SF-36: 36-item Short-Form Health Survey; IQR: interquartile range. \#: the assessment of respiratory discomfort used a visual scale, which combined numeric and analogic evaluation. The patients were asked to rate the intensity of their dyspnoea on a $10-\mathrm{cm}$ long ruler shaped like an arrow. It was bounded by " 0 : no respiratory discomfort" (the smallest base of the arrow) and to the right "10: maximal respiratory discomfort" (the head of the arrow). ": the adequacy of the prescribed durations of the NIV session and their actual durations was calculated as the sum of the actual duration of all NIV sessions per patient divided by the sum of their prescribed time; ${ }^{+}$: the Richmond Agitation-Sedation Scale (RASS) is a 10-point scale, ranging from -5 to +4 , where the lowest score ( -5 : unarousable) equals deeper sedation and the highest score (+4: combative) indicates major agitation; ${ }^{\S}$ : post-traumatic stress disorder (PTSD) related symptoms were assessed using the PDI scale. The PDI is a 13-item questionnaire, each item being scored from 0-4, with a higher score indicating a higher burden of PTSD-related symptoms; ${ }^{f}$ : NIV-associated discomfort, satisfaction and trauma were assessed with a $0-10$ numeric-visual scale at ICU discharge and a 0-10 numeric-verbal scale at day-90 (0: the absence of symptoms and -10: the maximum level of symptoms); \#\#: health-related quality of life (HRQoL) was measured using the physical and mental sub-scales of the SF-36 and HADS questionnaires. The SF-36 is a 36-item questionnaire scored from 0-100. The lower the score the more disability and the higher the score the less disability la score of 0 is equivalent to maximum disability and a score of 100 is equivalent to no disability). The HADS is a 14-item questionnaire, each item being scored from 0-3, with a higher score indicating a higher level of anxiety or depression la cut-off point of eight out of 21 identifies either anxiety or depression); ${ }^{\text {กा: }}$ vital status was assessed for 63 subjects at day- $90 ;{ }^{++}$: $p$-value for the comparison of the musical intervention group versus the control group; ${ }^{\S}$ : $p$-value for the comparison of the sensory deprivation group versus the control group. 
TABLE 3 Secondary outcomes: respiratory discomfort and physiological variables over time

\begin{tabular}{|c|c|c|c|c|c|c|}
\hline \multirow[t]{2}{*}{ Variable } & \multicolumn{3}{|c|}{ First NIV session } & \multicolumn{3}{|c|}{ All NIV sessions } \\
\hline & Slope value ${ }^{\#}{\text { units } \cdot \mathrm{h}^{-1}}^{-1}$ & $\begin{array}{l}\text { Change versus } \\
\text { control group }\end{array}$ & $\overline{p-v a l u e}$ & Slope value ${ }^{\#}$ units $\mathrm{h}^{-1}$ & $\begin{array}{l}\text { Change versus } \\
\text { control group }\end{array}$ & p-value \\
\hline \multicolumn{7}{|l|}{ Respiratory discomfort } \\
\hline Control group $(n=39)$ & $-0.26(-0.64 ; 0.12)$ & - & - & $-0.03(-0.14 ; 0.08)$ & - & - \\
\hline Musical intervention group ( $n=36$ ) & $-0.45(-0.83 ;-0.08)$ & -0.19 & 0.27 & $-0.14(-0.27 ;-0.01)$ & -0.11 & 0.21 \\
\hline Sensory deprivation group $(n=38)$ & $-0.23(-0.68 ; 0.22)$ & 0.031 & 0.30 & $-0.17(-0.31 ;-0.05)$ & -0.14 & 0.09 \\
\hline Musical intervention group $(n=36)$ & $-0.12(-0.62 ; 0.38)$ & -0.31 & 0.31 & $-0.19(-0.43 ; 0.05)$ & -0.21 & 0.21 \\
\hline Sensory deprivation group $(n=38)$ & $-0.21(-0.75 ; 0.32)$ & -0.40 & 0.21 & $-0.32(-0.59 ;-0.05)$ & -0.34 & 0.049 \\
\hline \multicolumn{7}{|l|}{ Transcutaneous oxygen saturation } \\
\hline Control group $(n=39)$ & $-0.18(-0.78 ; 0.41)$ & - & - & $0.26(-0.08 ; 0.60)$ & - & - \\
\hline Musical intervention group $(n=36)$ & $-0.27(-0.83 ; 1.23)$ & -0.08 & 0.89 & $-0.09(-0.48 ; 0.30)$ & -0.35 & 0.18 \\
\hline Sensory deprivation group $(n=38)$ & $0.20(-0.83 ; 1.23)$ & 0.39 & 0.52 & $0.2(-0.25 ; 0.65)$ & -0.06 & 0.83 \\
\hline \multicolumn{7}{|l|}{ Heart rate } \\
\hline Control group ( $n=39$ ) & $0.66(-1.07 ; 2.38)$ & - & - & $-0.41(-0.96 ; 0.15)$ & - & - \\
\hline Musical Intervention group ( $n=36$ ) & $-1.38(-3.16 ; 0.40)$ & -2.03 & 0.10 & $-0.19(-0.78 ; 0.41)$ & 0.22 & 0.59 \\
\hline Sensory deprivation group $(n=38)$ & $-0.01(-2.01 ; 1.99)$ & -0.67 & 0.62 & $-0.47(-1.11 ; 0.17)$ & -0.07 & 0.87 \\
\hline \multicolumn{7}{|l|}{ Systolic arterial pressure } \\
\hline Control group $(n=39)$ & $1.25(-1.32 ; 3.82)$ & - & - & $-0.17(-1.13 ; 0.78)$ & - & - \\
\hline Musical intervention group ( $n=36$ ) & $-2.40(-5.09 ; 0.29)$ & -3.65 & 0.05 & $-0.69(-1.73 ; 0.33)$ & -0.53 & 0.45 \\
\hline Sensory deprivation group $(n=38)$ & $1.63(-1.57 ; 4.83)$ & 0.39 & 0.85 & $-0.91(-1.98 ; 0.33)$ & -0.75 & 0.30 \\
\hline \multicolumn{7}{|l|}{ Mean arterial pressure } \\
\hline Control group (n=39) & $0.80(-0.55 ; 2.15)$ & - & - & $0.02(-0.57 ; 0.60)$ & - & - \\
\hline Musical intervention group ( $n=36$ ) & $-1.50(-3.00 ; 0.003)$ & -2.30 & 0.02 & $-0.42(-1.06 ; 0.21)$ & -0.45 & 0.30 \\
\hline Sensory deprivation group $(n=38)$ & $0.50(-1.35 ; 2.36)$ & -0.30 & 0.80 & $-0.47(-1.14 ; 0.20)$ & -0.50 & 0.27 \\
\hline
\end{tabular}

Data is presented as mean $(95 \% \mathrm{Cl}) .{ }^{\#}$ : slope values were obtained using a linear mixed-effects model fitted for each outcome. A positive slope means that the corresponding outcome increases over time, while a negative slope means that it decreases over time. The slope value of each intervention group (musical and sensory deprivation) was compared with the slope value of the control group using a Wald test.

end of the first NIV-session associated with the musical intervention, along with a significant reduction in the PDI on ICU discharge.

Musical intervention is gaining interest among non-pharmacological interventions $[15,24]$ and its easy implementation is a major argument for its generalisability. However, to the best of our knowledge, its benefits in improving NIV tolerance have never been assessed.

\begin{tabular}{lcccc}
\hline Group & \multicolumn{3}{c}{ Subjects } & Median (IQR) \\
\cline { 2 - 4 } & Total & Assessed & $\%$ & \\
\hline Control & 39 & 33 & 85 & $-1(-2 ; 0)$ \\
Sensory deprivation & 38 & 32 & 84 & $0(-2 ; 0)$ \\
Musical intervention & 36 & 32 & 89 & $-1(-3 ;-1)$ \\
All patients & 113 & 97 & 86 & $-1(-2 ; 0)$
\end{tabular}

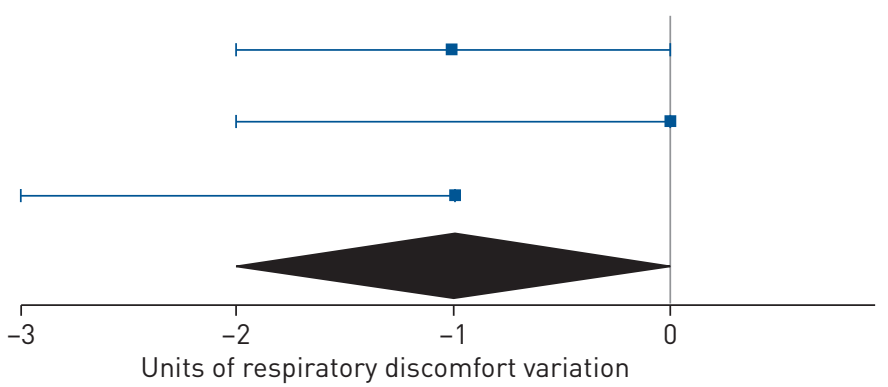

FIGURE 2 Median change in respiratory discomfort during the first noninvasive ventilation (NIV) session in the three groups. The median difference (IQR) was $-1(-2 ; 0)$ for the control group, $0(-2 ; 0)$ for the sensory deprivation group and $-1(-3 ; 1.1)$ for the musical intervention group ( $p=0.7$ by Wilcoxon rank-sum test). IQR: interquartile range. 
Our study failed to show a significant reduction in respiratory discomfort during NIV for ARF in ICU patients. Nevertheless, numerical values for the slopes of changes actually differed, with the musical intervention group's discomfort decreasing faster than the other groups (as shown in supplementary figure S1). Four major putative explanations for these results deserve discussion, as follows: 1) the levels of respiratory discomfort might have been only moderate at baseline (control group 4 (1-6); sensory deprivation group 5 (2-6); musical intervention group $5(1.5-7)$ ), which could have led to a "floor effect" (i.e. made it difficult to show an effect for the intervention); 2) all patients received NIV, which could in itself have led to an important respiratory improvement, thereby potentially masking the additional benefit of musical intervention; 3) the effect of musical intervention could have been negated by the additional sensory deprivation; 4) musical intervention could simply be ineffective.

Although the latter conclusion is "technically" valid given the negative results of the present trial, we believe that the more negative slope of changes in respiratory discomfort for the musical intervention group observed here and positive results from studies performed in other stressful situations suggest that other explanations may prevail $[15-17,25,26]$. Indeed, the three first conclusions may appear more plausible and are not mutually exclusive. Retrospectively, one may question the choice of respiratory discomfort as an outcome measure because it relates also to the patient's respiratory status and breathlessness. Hence, as mentioned above, the respective contribution of the intervention and of the treatment itself is difficult to decipher. Given the universally recognised major positive effect of NIV on breathlessness during ARF, one may hypothesise that NIV markedly improved patient comfort, especially after the first NIV session, to an extent that outgrew the effect of the musical intervention. Thus, it is possible that the added value of musical intervention may be more preeminent, in terms of NIV tolerance, for the subsequent sessions. Conversely, we felt that patients would have benefited "too much" from NIV and, to some extent, from aetiological treatments such as diuretics or $\beta_{2}$-agonists, had we focused on the subsequent NIV sessions. The first NIV session tended to be longer in the musical intervention group and this finding is in line with an increased tolerance of NIV in patients treated with musical interventions. A deeper insight is therefore needed with the assessment of other outcomes more likely to be influenced by music, such as anxiety for example.

Visual deprivation was, to a certain extent, unexpectedly poorly tolerated. The patients in the sensory deprivation group consistently reported a significantly lower degree of satisfaction related to NIV at day-90, reflecting a poor experience of NIV under these conditions. As previously [15], we considered it important to precisely assess the respective input of sensory deprivation and musical intervention in a potential alleviation of respiratory discomfort. Although the use of earplugs and eye masks has been shown to be effective in improving sleep quality in critically ill patients [27], compliance to sensory deprivation is reported to be low in ICU patients [18], with over 51\% rating use of eye masks' as "very uncomfortable" or "uncomfortable" [28]. On the basis of these arguments, taken altogether, we strongly believe that visual deprivation should not be used during NIV in the acute setting of respiratory distress.

We found that systolic and mean arterial pressure were significantly lower $1 \mathrm{~h}$ after NIV in patients receiving musical intervention in comparison with other groups. As the musical intervention lasted only $30 \mathrm{~min}$, we believe a noticeable remnant of a relaxing and anxiolytic effect from the musical intervention was present. A previous study [17] has described such an haemodynamic effect during musical intervention in invasively ventilated subjects, but with no information on its lasting effect. We show here that the lowering of BP is sustainable after the end of the musical intervention. A lower secretion of stress hormones in music-exposed patients might explain the mechanism involved in the lowering of BP [29]. A neuro-vegetative response, induced by the alleviation of stress and anxiety, might be the key to the lowering of cardiovascular parameters. As such, these findings shed light on potential mechanisms of action of music in humans.

In spite of a solid design, some limitations to the trial should be acknowledged. As discussed earlier, visual deprivation was poorly tolerated. One objection may be the absence of an assessment of delirium before enrolment, which might have biased the patients' self-reporting of respiratory discomfort. It has to be underlined that discussion with the patient in order to obtain written consent, as required by French legislation, was considered an acceptable screening for delirium. Moreover, no French version of the Confusion Assessment Method for the Intensive Care Unit existed at this time [30]. Next, this study could not be blinded, either on the patient's or the caregiver's side. However, the assessment of the primary endpoint was blinded from the treatment group, according to the Prospective Randomised Open-Blinded Endpoint (PROBE) method, by an independent member of staff who did not participate in conducting the NIV session. The choice of respiratory discomfort as the primary endpoint by itself might be questioned, as it is directly linked to patients' disease rather than to the therapies used. Moreover, as previously described for dyspnoea and pain [31], tolerance or comfort is a multidimensional experience and at least some of the dimensional variation results from different afferent mechanisms. It may be possible that music therapy may have a greater impact on other dimensions than the one explored in this study. 
On the one hand, the design of our trial might have hindered positive results, as follows: 1) the 30 min duration might have been too short to induce an effect on respiratory discomfort (i.e. an "under-dosed" music intervention); 2) the patient-directed music settings (type of music, volume etc) could have influenced these negative results. On the other hand, we wanted to perform a pragmatic trial and we acknowledge that it could have negatively biased the study. Lastly, the lack of the direct involvement of a music therapist in patients' care might be criticised. It is true that no music therapist intervened as a consultant at bedside. Nevertheless, these consultations are time- and labour-intensive. The advantage of the application used in our trial was that a skilled music therapist designed each musical piece and that his presence was not mandatory at bedside, allowing generalisability beyond the presence of a music therapist.

In conclusion, a musical intervention did not significantly reduce respiratory discomfort in patients requiring NIV for ARF. However, it did exert positive haemodynamic effects and reduce traumatic experience at ICU discharge. Notably, visual deprivation was poorly tolerated. This exploratory trial gives several clues with which to launch further studies aimed at determining the optimal settings for a musical intervention in this indication.

Acknowledgements: The authors wish to thank Stéphane Guétin (Chief Executive Officer of MUSIC CARE) who provided the music intervention software and Prof. Nicolas Terzi (Medecine Intensive et Réanimation, INSERM, Université Grenoble-Alpes, U1042, HP2, CHU Grenoble Alpes, Grenoble, France) for his insightful comments on the manuscript. We are indebted to the nurses and nurse-assistants of the three centres for their commitment to the study.

The MUS-IRA Investigators are as follows: Edwige Touré, Sandrine Monot, Damien Roux and Stephane Gaudry (Hôpital Louis Mourier); Lucile Leleu, Nathalie Piqueras, Lilia Berrahil-Meksen and Alain Gaffinel (Institut Gustave Roussy); Angélique Deschamps, Magalie Antoine, Aihem Yehia and Isabelle Vinatier (La Roche-sur-Yon District Hospital Center).

Conflict of interest: J. Messika reports congress fee reimbursement from Fisher and Paykel, outside the submitted work. J.-D. Ricard reports congress fee reimbursement from Fisher and Paykel, outside the submitted work.

Support statement: The sponsor was Assistance Publique-Hôpitaux de Paris (Dépt de la Recherche Clinique et de Développement). The study was funded by a grant from the Programme Hospitalier de Recherche Infirmière et Paramédicale (PHRIP 2013; Ministère de la Santé No. AOM 13-453). The funding source had no involvement in the study and no role in the writing of the manuscript or the decision to submit it for publication. Funding information for this article has been deposited with the Crossref Funder Registry.

\section{References}

1 Girou E, Brun-Buisson C, Taillé S, et al. Secular trends in nosocomial infections and mortality associated with noninvasive ventilation in patients with exacerbation of COPD and pulmonary edema. JAMA 2003; 290: 2985-2991.

2 Ram FSF, Picot J, Lightowler J, et al. Non-invasive positive pressure ventilation for treatment of respiratory failure due to exacerbations of chronic obstructive pulmonary disease. Cochrane Database Syst Rev 2004: CD004104.

3 Brochard L, Mancebo J, Wysocki M, et al. Noninvasive ventilation for acute exacerbations of chronic obstructive pulmonary disease. $N$ Engl J Med 1995; 333: 817-822.

4 L'Her E, Duquesne F, Girou E, et al. Noninvasive continuous positive airway pressure in elderly cardiogenic pulmonary edema patients. Intensive Care Med 2004; 30: 882-888.

5 Thille AW, Contou D, Fragnoli C, et al. Non-invasive ventilation for acute hypoxemic respiratory failure: intubation rate and risk factors. Crit Care 2013; 17: R269.

6 Antonelli M, Conti G, Moro ML, et al. Predictors of failure of noninvasive positive pressure ventilation in patients with acute hypoxemic respiratory failure: a multi-center study. Intensive Care Med 2001; 27: 1718-1728.

7 Delclaux C, L'Her E, Alberti C, et al. Treatment of acute hypoxemic nonhypercapnic respiratory insufficiency with continuous positive airway pressure delivered by a face mask: a randomized controlled trial. JAMA 2000; 284: 2352-2360.

8 Carlucci A, Richard JC, Wysocki M, et al. Noninvasive versus conventional mechanical ventilation. An epidemiologic survey. Am J Respir Crit Care Med 2001; 163: 874-880.

9 Schmidt M, Boutmy-Deslandes E, Perbet S, et al. Differential perceptions of noninvasive ventilation in intensive care among medical caregivers, patients, and their relatives: a multicenter prospective study-the PARVENIR study. Anesthesiology 2016; 124: 1347-1359.

10 Constantin JM, Perbet S, Futier E, et al. Impact de la sophrologie sur la tolérance des séances de ventilation non invasive chez des patients en insuffisance respiratoire aiguë [Impact of sophrology on non-invasive ventilation tolerance in patients with acute respiratory failure]. Ann Fr Anesth Reanim 2009; 28: 215-221.

11 Akada S, Takeda S, Yoshida Y, et al. The efficacy of dexmedetomidine in patients with noninvasive ventilation: a preliminary study. Anesth Analg 2008; 107: 167-170.

12 Clouzeau B, Bui HN, Vargas F, et al. Target-controlled infusion of propofol for sedation in patients with non-invasive ventilation failure due to low tolerance: a preliminary study. Intensive Care Med 2010; 36: $1675-1680$.

13 Rocco M, Conti G, Alessandri E, et al. Rescue treatment for noninvasive ventilation failure due to interface intolerance with remifentanil analgosedation: a pilot study. Intensive Care Med 2010; 36: 2060-2065.

14 Messika J, Kalfon P, Ricard J-D. Adjuvant therapies in critical care: music therapy. Intensive Care Med 2018; 44: 1929-1931. 
15 Chlan LL, Weinert CR, Heiderscheit A, et al. Effects of patient-directed music intervention on anxiety and sedative exposure in critically ill patients receiving mechanical ventilatory support: a randomized clinical trial. JAMA 2013; 309: 2335-2344.

16 Dijkstra BM, Gamel C, van der Bijl JJ, et al. The effects of music on physiological responses and sedation scores in sedated, mechanically ventilated patients. J Clin Nurs 2010; 19: 1030-1039.

17 Jaber S, Bahloul $\mathrm{H}$, Guetin S, et al. Effets de la musicothérapie en réanimation hors sédation chez des patients en cours de sevrage ventilatoire versus des patients non ventilés [Effects of music therapy in intensive care unit without sedation in weaning patients versus non-ventilated patients]. Ann Fr Anesth Reanim 2007; 26: 30-38.

18 Demoule A, Carreira S, Lavault S, et al. Impact of earplugs and eye mask on sleep in critically ill patients: a prospective randomized study. Crit Care 2017; 21: 284.

19 Messika J, Hajage D, Panneckoucke N, et al. Effect of a musical intervention on tolerance and efficacy of non-invasive ventilation in the ICU: study protocol for a randomized controlled trial (MUSique pour l'Insuffisance Respiratoire Aigue - Mus-IRA). Trials 2016; 17: 450.

20 Zigmond AS, Snaith RP. The hospital anxiety and depression scale. Acta Psychiatr Scand 1983; 67: 361-370.

21 Ware JE, Sherbourne CD. The MOS 36-item short-form health survey (SF-36). I. Conceptual framework and item selection. Med Care 1992; 30: 473-483.

22 Brunet A, Weiss DS, Metzler TJ, et al. The Peritraumatic Distress Inventory: a proposed measure of PTSD criterion A2. Am J Psychiatry 2001; 158: 1480-1485.

23 Jehel L, Brunet A, Paterniti S, et al. Validation de la version française de l'inventaire de détresse péritraumatique [Validation of the French version of the peritraumatic distress inventory]. Can J Psychiatry Rev Can Psychiatr 2005; 50: 67-71.

24 Bradt J, Dileo C. Music interventions for mechanically ventilated patients. Cochrane Database Syst Rev 2014: CD006902.

25 Hole J, Hirsch M, Ball E, et al. Music as an aid for postoperative recovery in adults: a systematic review and meta-analysis. Lancet 2015; 386: 1659-1671.

26 Bradt J, Dileo C, Magill L, et al. Music interventions for improving psychological and physical outcomes in cancer patients. Cochrane Database Syst Rev 2016: CD006911.

27 Hu R-F, Jiang X-Y, Chen J, et al. Non-pharmacological interventions for sleep promotion in the intensive care unit. Cochrane Database Syst Rev 2015: CD008808.

28 Richardson A, Allsop M, Coghill E, et al. Earplugs and eye masks: do they improve critical care patients' sleep? Nurs Crit Care 2007; 12: 278-286.

29 Chlan LL, Engeland WC, Savik K. Does music influence stress in mechanically ventilated patients? Intensive Crit Care Nurs 2013; 3: 121-127.

30 Chanques G, Garnier O, Carr J, et al. The CAM-ICU has now a French "official” version. The translation process of the 2014 updated Complete Training Manual of the Confusion Assessment Method for the Intensive Care Unit in French (CAM-ICU.fr). Anaesth Crit Care Pain Med 2017; 36: 297-300.

31 Banzett RB, O’Donnell CR, Guilfoyle TE, et al. Multidimensional dyspnea profile: an instrument for clinical and laboratory research. Eur Respir J 2015; 45: 1681-1691. 